\title{
Prophylactic and therapeutic parotidectomy for secondary neoplasms of the parotid gland
}

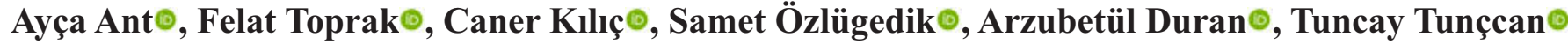

Department of Otorhinolaryngology, Head and Neck Surgery, University of Health Sciences, Dr. Abdurrahman Yurtaslan Ankara Oncology Training and Research Hospital, Ankara, Turkey

\section{ABSTRACT}

Objectives: The aim of this study was to review the demographic and clinicopathological findings of the patients who underwent prophylactic or therapeutic parotidectomy due to the secondary neoplasm of the parotid gland (SNP), the outcomes of the patients with SNP and to provide a perspective to the parotid and neck approach in head and neck skin cancer cases.

Methods: The clinical data of 178 patients who underwent parotidectomydue to neoplasm in the Department of Otorhinolaryngology, Head and Neck Surgery of a tertiary referral center from 2012 to 2017 were reviewed. Results: Among 178 patients, twenty-two (12.4\%) patients were found to underwentprophylactic or therapeutic parotidectomy due to SNP. Six of these patients had pathologically positive parotid metastases (therapeutic parotidectomy). The median age of six patients was 67 years (ranging from 2 to 83 years). Five patients were male and one was female. The most common indication of parotidectomy (prophylactic and therapeutic) and pathology of parotid mass due to SNP and Cutaneous Squamous Cell Carcinoma (SCC) (36.3\% and 50\%, respectively).

Conclusions: Owing to rapidly progressive feature and high morbidity, close observation of parotid in case of metastasis is crucial for recurrent SCC of the face and scalp.

Keywords: Head and neck neoplasms, neoplasm metastasis, parotid neoplasms

econdary neoplasm of the parotid gland (SNP) is $\checkmark$ a rare condition. Metastasis can both occur via lymphatic and hematogenous systems. The lymphatic system is the main route of head and neck tumors' metastases. The majority of these tumors are cutaneous malignancies of the scalp and face region [1]. The lymphatic system of the parotid space can be classified into three groups which are determined embryologically: The first group is intraparenchymal, the second group is intrafascial and extraparenchymal, and the last and third one is extrafacial and extraglandular such as the preauricular lymph nodes. The au- ricule, cheek, parietal scalp and ipsilateral side of the forehead are the primary sites that drain into the lymph nodes of the parotid space and through the deep cervical [1].

The thoracic duct and the Batson's paraspinal venous plexus were also reported to be the route of the distant metastases of breast, prostate, kidney and gastrointestinal carcinomas to the parotid gland [1]. However, the hematogenous system is the main route for the distant metastases of tumors such as sarcomas.

This study aimed to review the demographic and clinicopathological findings of the patients who under- 
went prophylactic or therapeutic parotidectomy due to SNP, the outcomes of the patients with SNP, and to provide a perspective to the parotid and neck approach in head and neck skin cancer cases.

\section{METHODS}

The clinical data of 178 patients who underwent prophylactic or therapeutic parotidectomy due to neoplasm in the Department of Otorhinolaryngology, Head and Neck Surgery (ORL-HNS) of a tertiary referral center from 2012 to 2017 were reviewed. The demographic and clinicopathological features of the patients with SNP were recorded.

All patients underwent superficial, total or radical parotidectomy as a part of the prophylactic, therapeutic and/or diagnostic purposeof a primary neoplasmother than the parotid gland. The finding asto the malignant mass or lymph node of the parotid gland with imaging studies such as US, CT, MRI and/or PET-CT and/or confirming fine needle aspiration cytologyor thru-cut aspiration biopsy (FNA, TCB); parotidectomy was added to the treatment protocol. En-block resection of the primary tumor with or without neck dissection was also performed for the tumors of head and neck other than nasopharynx carcinoma.

The indications of prophylactic or therapeutic parotidectomy due to SNP with subheadings can be assessed as:

\section{Squamous Cell Carcinoma (SCC) of the Skin}

In case of the parotid malignant mass finding with imaging studies and confirming FNA or TCB, the therapeutic parotidectomy was performed. In case of the local recurrence of the cutaneous SCC and Basosquamous Cell Carcinoma (BSCC)with one or more risk factors and/orsuspicious findings, lymph node of the parotid gland with imaging studies, the prophylactic parotidectomy was performed. Risk factors include tumor size more than $2 \mathrm{~cm}$, invasion into subcutaneous fat, poorly differentiated or metatypical or morpheophorm phenotype histopathology, high grade or desmoplasia, perineural invasion, lymphovascular invasion, location near parotid or lip, local recurrence, SCC in a preexisting scar, and immunosuppression [2].

\section{Malignant Melanoma (MM)}

The prophylactic, superficial parotidectomy was performed as a part of the sentinel lymph node biopsy (SLNB) with suspicious imaging findings. The therapeutic, radical parotidectomy was performed in one patient with intraoperative positive frozen biopsy for MM.

\section{Buccal and Lip (Oral Commissure) Carcinoma}

Buccal and Lip (oral commissure) Carcinoma that invade the skin (T4a) with suspicious imaging findings of the parotid; the prophylactic, superficial parotidectomy was performed with neck dissection and en-block tumor excision.

Lymphoma (in the follow-up period, after the previous treatment of lymphoma)

In case of the suspicious findings of FNA and PET-CT, the prophylactic (diagnostic), superficial parotidectomy was performed for the possibility of relapse.

\section{Nasopharynx Carcinoma (NPC)}

In case of the metastasis of NPC to the parotid gland that does not respond to the CRT treatment, the therapeutic, and total parotidectomy was performed.

\section{Rhabdomyosarcoma}

In case of the metastasis to the parotid space that does not respond to the CRT treatment, the therapeutic, total parotidectomy and tumor excision was performed.

According to the American Joint Committee on Cancer (AJCC) 2017, the malign neoplasms of the head and neck region were staged retrospectively. Neoadjuvant or adjuvant radiotherapy (RT) or chemoradiotherapy (CRT) was carried out, depending on the pathological evaluation of the surgical specimen includingthe primary tumor.

The approval was taken from the local institutional research committee (5417/4). The research was conducted in compliance with the Ethical Principles of the Declaration of Helsinki.

\section{Statistical Analysis}

In descriptive statistics related to continuous data; mean, standard deviation, median, minimum, maximum values; number and percentage values were 
given. The Kaplan-Meier method was used for survivor analysis of six patients with pathologically positive parotid metastasis.

\section{RESULTS}

Among 178 patients, 156 patients had primary tumors (134 benign, 22 malign tumors) of the parotid gland. Twenty-two $(12.4 \%)$ patients were found to underwentprophylactic or therapeutic parotidectomydue toSNP. Six of these patients had pathologically positive parotid metastases (therapeutic parotidectomy) and 18 underwent prophylactic parotidectomy. The SNP constituted 3.7\% (6/160) of all parotid neoplasms. The median age of six patientswas 67 years (ranging from 2 to 83 years). Five patients were male and one was female.

Among 22 patients underwent prophylactic or therapeutic parotidectomy; eight patients had SCC of the skin, two BSCC of the skin, four MM of the skin, two Buccal Carcinoma, one Lip (oral commissure) Carcinoma, two Lymphoma, one NPC, one Rhabdomyosarcoma.

\section{Squamous Cell Carcinoma of the Skin}

Eight $(36.3 \%)$ patients with SCC of the skin underwent parotidectomy: Five patients hadprophylactic, three patients had therapeutic parotidectomy. Six of the patients were male and two were female. The averageage of the patients was 70.6 years (range 21 to 87 years). The youngest one of these patients (21 years old) had Xeroderma Pigmentosum. Superficial, prophylactic parotidectomy was added to the treatment protocol of five patientswho had all of three risk factors liketumor size more than $2 \mathrm{~cm}$, recurrence, and location near parotid; as a result of the recurrence of the primary skin lesion. No pathological risk factor was reported as insufficient surgical margin, perineural or lymphovascular invasion in the first pathology reports of these patients. Parotid metastasis was not found in the surgical specimen of these five patients, pathologically.

In the remaining three patients with parotid mass and metastasis confirmed with the imaging studies and FNA, the parotid metastasis of SCC was found in the surgical specimen. One of these patients had a recurrenceone year after the surgery of the primarycutaneous zygoma lesion (pT2N0M0), as a parotid mass that invaded the facial nerve in the foramen stylomastoideum and caused total facial paralysis. Radical parotidectomy with radical neck dissection was performed. This patient presented intracranial metastasis in the follow-up period (six months later) and had died of thedisease. Another patient had parotid metastasis six months after the surgery of the primary cutaneous neck lesion (pT2N1M0). Total parotidectomy with radical neck dissection was performed. One year after the surgery, the patient presented iliac bone metastasis and

Table 1 . The patients with pathologically positive parotid metastasis

\begin{tabular}{|c|c|c|c|c|}
\hline No. & $\begin{array}{c}\text { Age } \\
\text { (years) }\end{array}$ & Gender & Location & $\begin{array}{l}\text { Histopathology } \\
\text { (TNM stage) }\end{array}$ \\
\hline 1 & 83 & Male & $\begin{array}{l}\text { The superficial and deep } \\
\text { lobe of the parotid }\end{array}$ & $\begin{array}{l}\text { SCC of zygoma skin } \\
\qquad\left(\mathrm{pT}_{2} \mathrm{~N}_{1} \mathrm{M}_{0}\right)\end{array}$ \\
\hline 2 & 81 & Male & $\begin{array}{l}\text { The superficial and deep } \\
\text { lobe of the parotid }\end{array}$ & $\begin{array}{l}\text { SCC of neck skin } \\
\quad\left(\mathrm{pT}_{2} \mathrm{~N}_{1} \mathrm{M}_{0}\right)\end{array}$ \\
\hline 3 & 67 & Female & $\begin{array}{l}\text { The superficial and deep } \\
\text { lobe of the parotid }\end{array}$ & $\begin{array}{l}\text { SCC of lateral canthal skin } \\
\qquad\left(\mathrm{pT}_{1} \mathrm{~N}_{1} \mathrm{M}_{0}\right)\end{array}$ \\
\hline 4 & 67 & Male & The deep lobe of the parotid & $\begin{array}{l}\text { SCC of nasopharynx } \\
\qquad\left(\mathrm{pT}_{2} \mathrm{~N}_{3} \mathrm{M}_{0}\right)\end{array}$ \\
\hline 5 & 65 & Male & $\begin{array}{l}\text { The superficial and deep } \\
\text { lobe of the parotid }\end{array}$ & $\begin{array}{l}\text { Malignant melanoma } \\
\text { (unknown primary tumor) }\end{array}$ \\
\hline 6 & 2 & Male & $\begin{array}{c}\text { The extraglandular region of } \\
\text { the parotid }\end{array}$ & $\begin{array}{l}\text { Rhabdomyosarcoma of the lower } \\
\text { limb }\end{array}$ \\
\hline
\end{tabular}


palliative CRT treatment was carried out. One year after the treatment, he died of thedisease. The third patient admitted to our clinic with grade 4 peripheral facial paralysis as a result of $2 \times 3 \mathrm{~cm}$ parotid mass, two years after the surgery of the primary cutaneous lateral canthal lesion (pT1N0M0). The infratemporal fossa surgery with radical parotidectomy was performed. Two years after the surgery, the patient presented intracranial metastasis and palliative CRT treatment was carried out. The common features of these three patients were the surgeries of the primary skin lesion were performed in another center and all of them had died of the disease (Table 1).

Adjuvant RT or CRT was carried out in all of the patients with skin SCC and parotid metastases.

\section{Basosquamous Cell Carcinoma of the Skin}

Two (9.1\%) patients with basosquamous cell carcinoma of the skin underwent parotidectomy with en-block tumor resection. Superficial, prophylactic parotidectomy was added to the treatment protocol of these two patients with risk factors like tumor size more than $2 \mathrm{~cm}$, recurrence and location near parotid; as a result of the localrecurrence of the primary skin lesion. No pathological risk factor was reported as insufficient surgical margin, perineural or lymphovascular invasion in the first pathology reports of these patients. No parotid metastasis was found in the surgical specimen of these patients.

\section{Malignant Melanoma}

Four (18.2\%) patients with MM underwent parotidectomy: One patient with evident parotid mass was misdiagnosed as a warthin tumor with FNA, preoperatively. As a result of the detection of the invaded facial nerve with parotid mass, frozen section biopsy was studied intraoperatively. After the diagnosis of MM with frozen section biopsy, radical parotidectomy with radical neck dissection was performed. The primary skin lesion was not found in this patient. Adjuvant CRT was carried out. No recurrence was detected in the second year of followup (Table 1).

In the remaining three patients, superficial, prophylactic parotidectomy was performed as a part of the sentinel lymph node biopsy (SLNB) with suspicious imaging findings. However, parotid metastasis was negative in the surgical specimen of the parotid. Two of these patients presented distant metastasis in the follow-up period. Palliative CRT was carried out and they died of thedisease.

\section{Buccal and Lip Carcinoma}

Superficial, prophylactic parotidectomy was performed in three $(13.6 \%)$ patients with two buccal and one lip (oral commissure) carcinoma with suspicious imaging findings. The stages of the tumor of these patients were pT4a, as a result of the invaded skin by the primary tumor. No parotid metastasis was found in the surgical specimen of these three patients. Selective or radical neck dissection with en-block tumor excision was also performed in these patients. In the follow-up period, one female patient with buccal carcinoma presented second primary neoplasm in the oral cavity as SCC of the tongue, four years later. After the treatment, this patient presented recurrence as tongue lesion and neck metastasis, she died of disease as the result of the neck metastasis that invaded the internal carotid artery. Adjuvant RT or CRT was carried out in all of these patients.

\section{Lymphoma}

Two $(9.1 \%)$ patients who were in the follow-up period after the treatment of lymphoma (one Hodgkin and one Marginal), presented with parotid mass. As the suspicious finding of FNA and PET-CT, superficial, prophylactic (diagnostic) parotidectomy was performed. No malignancy was found in the surgical specimen.

\section{Nasopharynx Carcinoma (NPC)}

Three years after the treatment of NPC with CRT, one $(4.5 \%)$ patient presented with a parotid mass in the deep lobe of the parotid gland, confirmed as NPC metastases with FNA preoperatively that do not respond to the CRT treatment. Total, therapeuticparotidectomy was performed. After the detection of multiple distant metastases in the followup period of two years, palliative CRT was carried out but the patient died of the disease (Table 1).

\section{Rhabdomyosarcoma}

One $(4.5 \%)$ patient who is two years old, presented with parotid space mass, six months after the treatment of the rhabdomyosarcoma of the lower limb. Total, therapeutic parotidectomy, and tumor 
excision were performed for the metastasis which was extrafacial and extraglandular that do not respond to the chemotherapy treatment. CRT was carried out after the surgery. No recurrence was detected in the followup period of two years (Table 1).

The Kaplan-Meier method showed that median survival time of six patients with pathologically positive parotid metastasis was 24 months (6-48). The cumulative survival rate in the first year was $66.7 \%$ (95\% confidence interval: $29-100 \%$ ).

\section{DISCUSSION}

Parotid gland tumors constitute $4-8 \%$ of the head and neck tumors. Malignancy rate for the parotid gland tumor is $0.2[3,4]$. SNPs havean average incidence of $6-8 \%$ of all parotid gland tumors, metastases originate from head and neck malignancies commonly and distant metastases rarely $[5,6]$. As a rare entity, there are few series of SNP and the literature mostly arises from the case reports. In our series, SNPs constituted $3.7 \%$ of all of the parotid gland tumors, the metastasis of head and neck malignancies were the most common pathology, compatible with the literature.

Rouviere [7] was first to declare the association of head and neck lymphatic drainage with the lymph nodes of parotid space in 1938. The parotid space takes the lymphatic drainage of the auricule, cheek, ipsilateral side of the forehead, parietal scalp and neck that are anterior and lateral to the parotid and drains into the internal jugular lymph nodes. The Cutaneous SCC of these anatomical regions is the most common pathology resulting in SNP. In our series, the most common indication of (prophylactic and therapeutic) parotidectomy and pathology of parotid mass due to SNP was Cutaneous SCC (36.3\%, 50\% respectively), consistent with the literature. As in our series, it characteristically involves the patients older than 65 years that have the primary tumor in the head and neck region [8]. In the presence of the parotid mass, preoperative FNA may affect the treatment decision with the assessment of malignancy and the distinction of primary and secondary neoplasms with high sensitivity and specificity [9]. It prepares the patient and the surgeon for a more extensive surgery such as radical parotidectomy and neck dissection, if needed [1]. In this series, there was only one false-negative case with a rare parotid metastasis of MM from an unknown primary.

The treatment of the Cutaneous SCC of the head and neck region is wide local excision of the lesion. The TNM classification based on AJCC 2017 has included prognostic risk factors that also give direction to the treatment approach (Tables 2 and 3) [10]. The recommended surgical margin of the lesion is $5 \mathrm{~mm}$ for minimal risk group that has $2 \mathrm{~mm}$ or lower vertical tumor thickness (VTT), $5-10 \mathrm{~mm}$ for the low-risk group (2.01-6 mm VTT), and $10 \mathrm{~mm}$ for the high-risk group (VTT $>6 \mathrm{~mm}$ ). As a footnote; the low-risk group with one or more risk factors should be managed as the high-risk group [11]. Insufficient surgical margin increases morbidity with the risk of local and regional recurrence. The small subset of Cutaneous SCC metastasizesto regional lymph nodes. In a systemic analysis, Cutaneous SCC of head and neck was reported to have $5 \%$ risk of regional metastasis [12]. In this context, the neck treatment is still a controversial issue. Elective neck dissection with parotidectomy for SCC with cN0 neck was recommended only for the risk of occult metastases

Table 2. The pathological tumor (pT) classification of Invasive Cutaneous Squamous Cell Carcinoma based on AJCC 2017 [10]

\begin{tabular}{lc}
\hline pT classification & Definition \\
\hline $\mathbf{p T}_{1}$ & Tumor $\leq 2 \mathrm{~cm}$ at largest horizontal width $+0-1$ high-risk feature \\
$\mathbf{p T}_{2}$ & $\begin{array}{r}\text { Tumor } \leq 2 \mathrm{~cm} \text { at largest horizontal width }+2-5 \text { high-risk features or } \\
\text { tumor }>2 \mathrm{~cm} \text { at largest horizontal width }\end{array}$ \\
$\mathbf{p T}_{3}$ & Infiltration of facial and cranial bones \\
$\mathbf{p T}_{\mathbf{4}}$ & Infiltration of skeletal bone or skull base \\
\hline $\mathrm{pT}=$ pathological tumor &
\end{tabular}




\begin{tabular}{|c|c|c|}
\hline Prognostic Risk Factors & Low Risk Factors & High Risk Factors \\
\hline Tumor diameter & Less than $2 \mathrm{~cm}$ & More than $2 \mathrm{~cm}$ \\
\hline Location & $\begin{array}{l}\text { Sun exposed sites } \\
\text { (except ear/lip) }\end{array}$ & $\begin{array}{c}\text { Ear/lip } \\
\text { Non sunexposed sites } \\
\text { SCC arising in radiation sites, scars, burns } \\
\text { or chronic inflammatory conditions } \\
\text { Recurrent SCC }\end{array}$ \\
\hline Depth/level of invasion & $\begin{array}{l}\text { Less than } 6 \mathrm{~mm} / \text { invasion } \\
\text { above subcutaneous fat }\end{array}$ & $\begin{array}{c}\text { More than } 6 \mathrm{~mm} / \text { invasion beyond } \\
\text { subcutaneous fat }\end{array}$ \\
\hline Histologic features & $\begin{array}{l}\text { Well differentiated grade } \\
\text { Common variant or } \\
\text { verrucous }\end{array}$ & $\begin{array}{c}\text { Moderately, or poorly differentiated grade } \\
\text { Acantholytic, spindle, or desmoplastic } \\
\text { subtype } \\
\text { Perineural invasion }\end{array}$ \\
\hline Surgical margins & Clear & Incomplete excision \\
\hline Immune status & Immunocompetent & $\begin{array}{c}\text { Immunosuppressed } \\
\text { (organ transplant recipients, chronic } \\
\text { immunosuppressive disease or treatment) }\end{array}$ \\
\hline
\end{tabular}

more than $19 \%$ as the classical knowledge [13]. D'Souza and Clark [2] defined clinical and pathological risk factors (noted in the methods section) to determine the patients who have a higher risk of regional metastases. The temporal region, cheek, auricle, and forehead are the most common sites of Cutaneous SCC that metastasize to the parotid gland [14]. As a multicenter study, Andruchow et al. [15] reported the rate of parotid involvement as high as $80 \%$ for Cutaneous SCC. Kadakia et al. [16] reported $25 \%$ of occult parotid metastases rate in the patients with Cutaneous SCC of Temporal Region of at least 2 $\mathrm{cm}$ and recommended prophylactic parotidectomy especially for tumors with the perineural or vascular invasion at the primary site.

Considering the low cure rate of salvage surgery, increasing distant metastasis risk of loco-regional involvement, the high morbidity of $\mathrm{N}+$ neck dissection; the morbidity of parotidectomy and neck dissection (if necessary) may be thought to be negligible [14, 17]. However, Hoch et al. [18] and Osborne et al. [19] recommended against prophylactic parotidectomy, who studied Cutaneous SCC of head and auricle, respectively. These patients are also recommended to be screened with parotid MRI for infraclinical metastases in the Guidelines of the French
Society of ORL [20].

In this study, prophylactic parotidectomy was added to the treatment protocol of five patients with recurrent SCC and two patients with recurrent BSCC that had two or more risk factors (commonly tumor size more than $2 \mathrm{~cm}$, recurrence and location near parotid), as the clinical preference. No parotid metastasis was found in the surgical specimen of these patients. There was no local or regional recurrence in these cases in the follow-up period.

Tumors located on the scalp and face anterior to the vertical line drawn through the external auditory canal commonly spread to the parotid and through upper cervical nodes; parotidectomy should be considered as a part of the neck dissection. The incidence of occult neck metastasis in the presence of parotid metastasis was reported as between $29-35 \%$ in the literature $[1,21]$. For the management of cutaneous SCC with parotid metastasis, a combined modality treatment including neck dissection with parotidectomy and RT is recommended as in our series [22]. The survival rates are low in our series, as all of the patients had died of the disease in 6-24 months after the treatment, compared to other series [23, 24]. In this context, loco-regional treatment and close follow-up are crucial for these patients. 
SCC of the oral cavity and oropharynx were also reported as the primary sites of the parotid metastases $[4,5,25,26]$. Increased number of cancer-positive lymph nodes were reported to increase the risk of parotid involvement by Harada et al. [27]. The skin involvement of SCC of the oral cavity and oropharynx were also considered to increase the possibility of metastasis to the parotid gland. In the present series, no pathologically positive metastases were assessed in the parotid specimens. In the presence of advanced imaging methods, parotidectomy with suspicious findings is questionable. However, the facial nerve involvement and facial paralysis occurrence are seen to be rapidly progressive in these patients with parotid metastases. Also, these cases require extensive treatment or become unsalvageable compared with low morbidity of parotidectomy.

The parotid gland contains an average of 5-10 lymph nodes as distinct from other salivary glands. Lymphoproliferative diseases such as lymphoma may involve lymph nodes in the parotid gland and also glandular parenchyma [28]. As the low diagnostic value of FNA for lymphoproliferative diseases, excisional lymph node biopsy as superficial partial parotidectomy was performed to the patients who were cured and in the follow-up period of lymphoma. These were pathologically negative for lymphoma. In this regard, TCB might be an alternative method to the excisional lymph node biopsy for the diagnosis.

In NPC, parotid metastasis may occur via retropharyngeal lymph nodes, indirectly through periparotid extension, retrograde extension from massive neck metastasis and rarely skip metastasis $[29,30]$. Parotid lymph node (PLN) sparing intensitymodulated radiotherapy (IMRT) to reduce the side effect of xerostomia was reported to cause $2.8 \%$ of PLN metastasis after IMRT. PLN metastasis of NPC was recommended to assess as N3 and treat with aggressive concurrent chemoradiotherapy to decrease the possibility of distant metastasis. Parotidectomy is a choice for treatment-resistant (CRT) cases, as the cases with NPC and Rhabdomyosarcoma in the present series [31-33].

, the distant metastases of the upper aerodigestive tract, lung, breast, pancreas, and colon cancers to the parotid gland have been reported in the literature. Hematogenous dissemination is the main route for these cancer types $[4,5,10,34]$. Rhabdomyosarcoma is extremely rare as metastatic disease to the parotid space. There was only one case with parotid, auricle and lung metastases in the literature that had died of disease 14 months after parotidectomy [1]. In the present series, two years old patient had no distant metastases other than parotid space and he has been in the follow-up period of 24 months after parotidectomywithout recurrence.

\section{CONCLUSION}

Although secondary neoplasms of the parotid gland are uncommon, it should be taken into consideration for the differential diagnosis of the mass of the parotid gland.Owing to rapidly progressive feature and high morbidity, close observation of parotid in case of metastasis is also crucial for recurrent SCC of the face and scalp. The larger number of patients is needed to recommend a therapeutic approach.

\section{Conflict of interest}

The authors disclosed no conflict of interest during the preparation or publication of this manuscript.

\section{Financing}

The authors disclosed that they did not receive any grant during conduction or writing of this study.

\section{REFERENCES}

1. Nuyens M, Schupbach J, Stauffer E, Zbaren P. Metastatic disease to the parotid gland. Otolaryngol Head Neck Surg 2006; 135:844-8.

2. D'Souza J, Clark J. Management of the neck in metastatic cutaneous squamous cell carcinoma of the head and neck. Curr Opin Otolaryngol Head Neck Surg 2011;19:99-105.

3. Lukšić I, Virag M, Manojlović S, Macan D. Salivary gland tumours: 25 years of experience from a single institution in Croatia. J Craniomaxillofac Surg 2012;40:e75-81.

4. Barnes L, Eveson JW, Reichart P, Sidransky D. (Eds.): World Health Organization Classification of Tumours. Pathology and Genetics of Head and Neck Tumours. IARC Press: Lyon, 2005. 5. Franzen AM, Günzel T, Lieder A. Parotid gland metastasesof distant primary tumours: a diagnostic challenge. Auris Nasus Larynx 2015;29:187-91.

6. Pomar Blanco P, Martín Villares C, San Román Carbajo J, Tapia Risueño M, Fernández Pello M. [Metastasis to the parotid gland]. Acta Otorrinolaryngol Esp 2006;57:47-50. [Article in 
Spanish]

7. Rouviere H. Anatomy of the Human Lymphatic System (Tobias JM, translator.) Ann Arbor, Edward Brothers, 1938.

8. Schroeder WA, Stahr WD. Malignant neoplastic disease of the parotid lymph nodes. Laryngoscope 1998;108:1514-9.

9. Zbaren P, Schar C, Hotz MA, Loosli H. Value of fine-needle aspiration cytology of parotid gland masses. Laryngoscope 2001;111:1989-92.

10. Lydiatt WM, Patel SG, O'Sullivan B, Brandwein MS, Ridge JA, Migliacci JC, et al. Head and neck cancers - major changes in the American Joint Committee on cancer eighth edition cancer staging manual. CA Cancer J Clin 2017;67:122-37.

11. Stratigos A, Garbe C, Lebbe C, Malvehy J, Del Marmol V, Pehamberger $\mathrm{H}$, et al. Diagnosis and treatment of invasive squamous cell carcinoma of the skin: European consensus-based interdisciplinary guideline. Eur J Cancer 2015;51:1989-2007.

12. Jambusaria-Pahlajani A, Miller CJ, Quon H, Smith N, Klein RQ, Schmults CD. Surgical monotherapy versus surgery plus adjuvant radiotherapy in high-risk cutaneous squamous cell carcinoma: a systematic review of outcomes. Dermatol Surg 2009;35:574-85.

13. Wong WK, Morton RP. Elective management of cervical and parotid lymph nodes in stage N0 cutaneous squamous cell carcinoma of the head and neck: a decision analysis. Eur Arch Otorhinolaryngol 2014;271:3011-9.

14. Skulsky SL, O'Sullivan B, McArdle O, Leader M, Roche M, Conlon PJ, et al. Review of high-risk features of cutaneous squamous cell carcinoma and discrepancies between the American Joint Committee on Cancer and NCCN Clinical Practice Guidelines in Oncology. Head Neck 2017;39,578-94. 15. Andruchow JL, Veness MJ, Morgan GJ, Gao K, Clifford A, Shannon KF, et al. Implications for clinical staging of metastatic cutaneous squamous carcinoma of the head and neck based on a mulitcenter study of treatment outcomes. Cancer 2006;106:107883.

16. Kadakia S, Saman M, Gordin E, Marra D, Ducic Y. The role of parotidectomy in the treatment of auricular squamous cell carcinoma. Otolaryngol Head Neck Surg 2015;152:1048-52.

17. Haksever M, Akduman D, Demir M, Aslan S, Yanılmaz M, Solmaz F. The treatment of neck and parotid gland in cutaneous squamous cell carcinoma of face and forehead and the review of literature. Ann Med Surg 2015;4:48-52.

18. Hoch S, Franke N, Katabi N, Werner JA, Teymoortash A. The value of elective parotidectomy in advanced squamous cell carcinoma of the skin of the head. Anticancer Res 2014;34:24336.

19. Osborne RF, Shaw T, Zandifar H, Kraus D. Elective parotidectomy in the management of advanced auricular malignancies. Laryngoscope 2008;118:2139-45.

20. Durbec M, Couloigner V, Tronche S, Albert S, Kanitakis J, Ltaief Boudrigua A, et al. Guidelines of the French Society of
Otorhinolaryngology (SFORL), short version. Extension assessment and principles of resection in cutaneous head and neck tumors. European Ann Otorhinolaryngol Head Neck Dis 2014; $131: 375-83$.

21. O'Brien CJ, McNeil ED, McMahon JD, Pathak I, Lauer CS. Incidence of cervical node involvement in metastatic cutaneous malignancy involving the parotid gland. Head Neck 2001;23:744-8.

22. Taylor BW, Brant TA, Mendenhall NP, Mendenhall WM, Cassisi NJ, Stringer SP, et al. Carcinoma of the skin metastatic to parotid area lymph nodes. Head Neck 1991;13:427-33.

23. Bron LP, Traynor SJ, McNeil EB, O’Brien CJ. Primary and metastatic cancer of the parotid: comparison of clinical behaviour in 232 cases. Layngoscope 2003;113:1070-5.

24. Chua MS, Veness MJ, Morgan G, Shakespeare T, Hehir A, Gebski V, et al. Parotid lymph node metastasis from cutaneous squamous-cell carcinomas: treatment outcome and prognostic factors following surgery and adjuvant radiotherapy. Australas Radiol 2002;46:174-9.

25.PastoreA, Ciorba A, Soliani M, Di Laora A, Valpiani G, Bianchini C, et al. Secondary malignant tumors of the parotid gland: not a secondary problem. J BUON 2017;22:513-8.

26. Pisani P, Krengli M, Ramponi A, Guglielmetti R, Pia F. Metastases to parotid gland from cancers from upper airway and digestive tract. Br J Oral Maxillofacial Surg 1998;36:54-7.

27. Harada H, Omura K. Metastasis of oral cancer to the parotid node. Eur J Surg Oncol 2009;35:890-4.

28. Zhang C, Cohen JM, Cangiarella JF, Waisman J, McKenna BJ, Chhieng DC. Fine-needle aspiration of secondary neoplasms involving the salivary glands. Am J Clin Pathol 2000;113:21-8.

29. Gregoire V, Ang K, Budach W. Delineation of the neck node levels for head and neck tumors: a 2013 update. DAHANCA, EORTC, HKNPCSG, NCIC CTG, NCRI, RTOG, TROG consensus guidelines. Radiother Oncol 2014;110:172-81.

30. Dong XR, Zhang T, Fan L, Zhang S, Wu G. Parotid gland metastasis of nasopharyngeal carcinoma: case report and review of the literature. J Int Med Res 2009;37:1994-9.

31. Yeung KW, Chiang PT, Chang CY, Yuan BC. A parotid gland mass as an initial metastatic manifestation of nasopharyngeal carcinoma. J Cancer Res Pract 2018;5:123-6.

32. Wang S, Lou J, Zhang S, Guo L, Wang K, Ge M. Metastasis of nasopharyngeal carcinoma to parotid lymph nodes: a retrospective study. World J Surg Oncol 2015;13:1.

33. Xu Y, Chen X, Zhang M, Xiao Y, Zong J, Guo Q, et al. Prognostic effect of parotid area lymph node metastases after preliminary diagnosis of nasopharyngeal carcinoma: a propensity score matching study. Cancer Med 2017;6:2213-21.

34. Dequanter D, Lothaire P, Andry G. Secondary malignant tumors of the parotid. Ann Otolaryngol Chir Cervicofac 2005;122:18-20. 